Revista Energia na Agricultura

ISSN 1808-8759

\title{
MODELO MATEMÁTICO PARA DETERMINAÇÃO DO CUSTO E PRODUÇÃO DE ENERGIA NA CULTURA DA CANA-DE-AÇÚCAR ${ }^{1}$ RÔMULO PIMENTEL RAMOS ${ }^{2} \&$ HELENICE DE OLIVEIRA FLORENTINO SILVA ${ }^{3}$
}

RESUMO: A cana-de-açúcar possui grande importância social e econômica para o brasileiro. O Brasil é maior produtor de cana-de-açúcar do mundo, com a produção foi de 597,8 milhões de toneladas na safra de 2009/2010, uma alta de 4,4\% em relação as 571,40 milhões de toneladas colhidas em 2008/2009. Por outro lado, o crescimento acelerado deste setor tem trazido problemas de grandes dimensões e dificultado o gerenciamento das empresas, estes problemas são de ordens estruturais e ambientais. Desta forma é importante conhecer todo processo de cultivo da cana, que vai desde o preparo do solo até processo o industrial, que ocorre da recepção da cana até a saída dos produtos, e buscar formas de minimizar os custos de produção. Assim, surge a necessidade de desenvolvimento de ferramentas que auxiliem os gestores das empresas nas tomadas de decisões. A modelagem matemática pode ser utilizada como uma importante fonte de produção de estimativas para este setor, facilitando cálculos e auxiliando as decisões. O objetivo deste trabalho foi estudar toda a cadeia produtiva da cana e investigar modelos matemáticos para estimar quantitativamente o balanço de energia e o custo de produção do processo de produção da cana. No presente trabalho foi desenvolvido um modelo matemático e uma planilha para estimar o custo do processo produtivo da cana de açúcar, permitindo determinar qual a área, em hectare, que cada variedade de cana deve ser plantada de forma a obter o menor custo possível para o processo de produção da cultura da canade-açúcar. Para a área com colheita manual o modelo determinou três variedades: SP80-1816, SP80-3280 e RB855113, e para a área de colheita mecanizada foram determinadas quatro variedades: SP80-1816, SP80-3280, RB855536 e SP70-1143. O modelo de programação linear proposto para minimização de custos, juntamente com a planilha implementada, mostram-se ferramentas promissoras para auxiliar a gestão das empresas do setor sulcroalcooleiro.

Palavras-chave: Cana-de-açúcar, otimização, minimização de custo.

\footnotetext{
${ }^{1}$ Parte da dissertação de mestrado do primeiro autor intitulada: Modelo matemático para custo e energia na produção de açúcar e álcool.

${ }^{2}$ Aluno do Programa de Pós-graduação em Agronomia - Energia na Agricultura, FCA/UNESP, Botucatu/SP. End: Rua Antonio Nunes da silva Sobrinho, 870. CEP: 18610-170. Botucatu/SP. e-mail: romulo.pimentel@ hotmail.com

${ }^{3}$ Orientadora e docente do Departamento de Bioestatística, Instituto de Biociências UNESP, Botucatu/SP - Brasil; End: Rubião Junior. CEP: 18618-000. Botucatu/SP. Fone: 14-3811 6272. e-mail: helenice@ibb.unesp.br
} 


\section{MATHEMATICAL MODEL FOR DETERMINING THE ENERGY COST AND PRODUCTION OF CULTIVATING SUGARCANE}

SUMMARY: Sugarcane has a great social and economic importance for Brazil. Brazil is the largest producer of sugarcane in the world. In 2009/10 crop production was 597.8 million tons, up 4.4\% compared to 571.40 million tons harvested in 2008 /09. On the other hand, the accelerated growth of this sector has brought about a number of larger problems from structural and environmental orders making the management of companies more difficult. Thus it is important to know the entire process of producing sugarcane from soil preparation to industrialization that being the reception of the sugarcane until its output as a finished product to seek ways to minimize production cost. This is proving the need of developing tools that will assist managers in corporate decision making. The mathematical modeling can be utilized with an important source of production estimates for this sector, facilitating calculations and aiding in decisions. The objective of this work was to study the entire production chain of sugarcane and investigate mathematical models to estimate quantitatively the energy balance and production cost of the production phase of sugarcane. We developed a mathematical model and spreadsheet to estimate the cost of the sugarcane production process to determine which area in hectares that each variety of cane should be planted in order to obtain the lowest possible cost in the production process. For the area using manual harvesting the model was determined using three varieties: SP80-1816, SP80-3280 and RB855113, also the area of mechanical harvesting has been determined by using four varieties: SP80-1816, SP80-3280, RB855536 and SP70-1143. The linear programming model proposed to minimize costs along with a spreadsheet that has been implemented in this work shows promising tools to assist with the management of companies in the industry.

Keywords: Sugarcane, optimization, cost minimization.

\section{INTRODUÇÃO}

O setor sucroalcooleiro consolidou-se ao longo dos últimos anos como um dos pilares da atividade agrícola brasileira. A expressiva expansão da cana-de-açúcar frente a outras culturas, tais como a soja, o milho e mesmo a pecuária, demonstra a dimensão que o setor possui dentro do cenário nacional. Da mesma forma, observa-se grande crescimento dos produtos gerados a partir desta matéria prima, sendo eles: o álcool, o açúcar e a energia elétrica (MARQUES, 2009). 
Para tanto somente as vendas de bens finais (etanol, açúcar, bioeletricidade, levedura e aditivo e crédito de carbono, entre outros) representam 1,5\% do PIB nacional, ou US\$28,1 bilhões. O País, responsável por $50 \%$ das exportações mundiais de açúcar, pode chegar a $60 \%$ deste montante em cinco anos (USP, 2009).

A capacidade produtiva e os baixos custos relativos ao setor sucroalcooleiro no Brasil vêm chamando a atenção das principais economias do mundo. O potencial agrícola brasileiro é incontestável, porém, ainda existem muitas divergências no que se refere aos custos de produção sucroalcooleira, uma vez que são poucos os trabalhos divulgados e de domínio público sobre o tema, além da falta de uma metodologia de aferição de custos, comum às diversas unidades industriais. Estes trabalhos poderiam trazer muitos benefícios ao setor quando acompanhados de prática de gestão de custos nas empresas, desenvolvimento de políticas públicas, aprimoramento da forma de pagamento da cana de açúcar, entre outros (MARQUES, 2009).

Além dos poucos trabalhos públicos, a complexidade desta área tem dificultado os gestores das empresas sucroalcooleiras e, toda pesquisa voltada ao auxílio das administrações deste setor, tem sido muito bem recebida.

Desta forma, neste trabalho se buscou conhecer todo o processo de cultivo da cultura da cana-deaçúcar e seus custos, que vai desde o preparo do solo até o processo industrial, que ocorre da recepção da matéria-prima até a saída dos produtos, e buscar alternativas de minimizar esses custos de produção. Assim sendo, surge a necessidade de desenvolvimento de ferramentas que auxiliem na minimização dos custos e ajude os gestores nas tomadas de decisões. Desta forma, a modelagem matemática pode ser utilizada como uma importante ferramenta para auxiliar na produção de estimativas para este setor, facilitando cálculos e tomada de decisões.

Alguns trabalhos envolvendo modelos matemáticos, de custo e balanço de energia da cana-deaçúcar, são encontrados na literatura, porém, muitos deles, só envolvendo o processo de recolhimento do palhiço, assim como os desenvolvidos por Florentino (2005) e Tolentino e Florentino (2008), onde eles propõem um modelo que, escolhem a variedade de cana-de-açúcar de forma a maximizar o balanço de energia e minimize o custo de recolhimento do palhiço. Florentino et al. (2008), também propõe um modelo para auxiliar na escolha da variedade de cana de forma a minimizar os custos do processo de transferência da biomassa residual da colheita.

O objetivo deste trabalho é estudar toda a cadeia produtiva da cana-de-açúcar e investigar modelos matemáticos para estimar quantitativamente o balanço de energia e o custo de produção da cultura, desde o seu plantio até a entrega da matéria-prima na usina. 


\section{MATERIAL E MÉTODOS}

Foram realizadas consultas a literaturas disponíveis, para entendimento de todo o processo de produção da cana-de-açúcar, desde o plantio até entrega na usina, levantamento de fatores que influenciam o custo e investigação de modelos matemáticos para o cálculo do custo para todo o sistema produtivo, levantados os dados de custos para cada fase do processo. Estes dados foram organizados em tabelas, utilizando o aplicativo computacional Microsoft Excel, no Laboratório Científico de Informática (LCI) do Departamento de Bioestatística do Instituto de Biociências da UNESP, campus de Botucatu.

Foi desenvolvida, neste trabalho, uma planilha para cálculo dos custos de preparo de solo e plantio da cultura, assim como o custo de colheita da cana. O custo foi calculado para os dois tipos de colheita: manual e mecanizada, sendo o custo total de colheita a soma destes. Na colheita mecanizada, tem-se também o custo de recolhimento do palhiço.

Para estimar estes custos, inicialmente são selecionadas todas as variedades adaptáveis ao solo local, com suas respectivas produtividades e posteriormente resolve-se o modelo proposto. Este modelo indicará quais as variedades de cana serão plantadas e a área destinada para este plantio. O modelo é aplicado ao tipo de colheita requerida pelo produtor: manual com queima e/ou mecanizada.

Definida a área de plantio de cada variedade, a planilha calcula automaticamente todos os custos do processo, que vai desde a produção e entrega da matéria-prima na indústria.

Para a parte energética, foi calculado o consumo de energia não renovável, ou seja, consumo de óleo diesel de cada operação da produção da cana, sendo em seguida transformado em megajoule (MJ). Para a energia produzida pelo palhiço utilizou-se a equação (2.1) e para o gasto de energia do recolhimento do palhiço, utilizou-se a equação (2.2).

A energia da biomassa residual (EB) de colheita da variedade i plantada no talhão j, EBij (MJ) é calculada pela fórmula:

$$
\mathrm{EB}_{\mathrm{ij}}=\mathrm{Ec}_{\mathrm{Bi}} \mathrm{P}_{\mathrm{Bi}} \mathrm{L}_{\mathrm{j}}
$$

Sendo, $\mathrm{Ec}_{\mathrm{Bi}}$ a estimativa da energia calorífica gerada por uma tonelada do resíduo de colheita da variedade $\mathrm{i}\left(\mathrm{MJ} \mathrm{t}^{-1}\right) ; \mathrm{P}_{\mathrm{Bi}}$ a estimativa da massa de palhiço gerada por um hectare de cana da variedade $\mathrm{i}$, em $t_{\text {ha }}{ }^{-1}$, e $\mathrm{L}_{\mathrm{j}}$ a área do talhão $\mathrm{j}$ (ha).

A energia gasta no processo de transferência (ET) da biomassa da variedade i, plantada no talhão $\mathrm{j}$, para o centro de processamento $\left(\mathrm{ET}_{\mathrm{Bij}}\right)$, é dada pela soma da energia utilizada para enleirar e compactar $\left(\mathrm{E}_{\mathrm{EC} \mathrm{ij}}\right)$, carregar $\left(\mathrm{E}_{\mathrm{Cij}}\right)$ e transportar $\left(\mathrm{E}_{\mathrm{Tij}}\right)$ esta biomassa. 


$$
\mathrm{ET}_{\mathrm{Bij}}=\mathrm{E}_{\mathrm{ECij}}+\mathrm{E}_{\mathrm{Cij}}+\mathrm{E}_{\mathrm{Tij}}
$$

\subsection{Modelo para custo}

Para atender o objetivo desse trabalho, desenvolveu-se um modelo matemático para estimar o custo do processo produtivo da cana de açúcar e auxiliar na gestão das usinas e produtores, que consiste em determinar qual a área, em hectare, que cada variedade de cana deve ser plantada, de forma a obter o menor custo possível para o processo de produção da cana, desde o preparo do solo até a entrega da cana na usina.

Para formulação do modelo, a área para plantio foi dividida em duas partes, de acordo com a necessidade da usina: uma parte para plantio da cana que deverá ser queimada na pré-colheita e outra para cana que será colhida sem queima. Esta divisão se fez necessária, pois os custos são diferentes para cada tipo de colheita. Na colheita de cana queimada têm-se os custos de aceiro, queima, corte manual, carregamento da cana para o caminhão e transporte da cana do campo para a usina. Na colheita mecanizada têmse os custos de corte e transporte da cana do campo para a usina.

\subsubsection{Modelo para cana-de-açúcar com colheita manual}

Denotando $\mathrm{CQ}_{\mathrm{i}}$ o custo de colheita por hectare da cana de variedade i tem-se que para cana a ser queimada, $\mathrm{CQ}_{\mathrm{i}}$ é calculado da seguinte forma:

$$
\mathrm{CQ}_{\mathrm{i}}=\mathrm{C}_{\mathrm{ai}}+\mathrm{C}_{\mathrm{qi}}+\mathrm{C}_{\mathrm{coi}}+\mathrm{C}_{\mathrm{cai}}+\mathrm{C}_{\mathrm{ti}}
$$

Onde:

$\mathrm{C}_{\mathrm{ai}}$ é o custo do aceiro da cana de variedade i $\left(\mathrm{R} \$ \mathrm{ha}^{-1}\right)$;

$\mathrm{C}_{\mathrm{qi}}$ é o custo da queima da cana de variedade i $\left(\mathrm{R} \$ \mathrm{ha}^{-1}\right)$;

$\mathrm{C}_{\mathrm{coi}}$ é o custo do corte da cana de variedade i $\left(\mathrm{R} \$ \mathrm{ha}^{-1}\right)$;

$\mathrm{C}_{\text {cai }}$ é o custo do carregamento da cana de variedade i $\left(\mathrm{R} \$ \mathrm{ha}^{-1}\right)$;

$\mathrm{C}_{\mathrm{ti}}$ é o custo de transporte da cana de variedade i $\left(\mathrm{R} \$ \mathrm{ha}^{-1}\right)$.

Denotando por $\mathrm{X}_{\mathrm{i}}$ a variável do modelo que fornecerá a área (ha) em que deverá ser plantada a cana-de-açúcar de variedade i, propõe-se o modelo:

$$
\min \sum_{i=1}^{n} C Q_{i} X_{i}
$$

sujeito a 


$$
\begin{aligned}
& \sum_{i=1}^{n} X_{i}=A Q \\
& \sum_{i=1}^{n} P_{i} X_{i} \geq P \\
& F I \leq \sum_{i=1}^{n} F_{i} X_{i} \leq F S \\
& 0 \leq X_{i} \leq V, \mathrm{i}=1, \ldots, \mathrm{n} .
\end{aligned}
$$

onde,

$\mathrm{i}=1,2, \ldots, \mathrm{n}$ são os índices que representam as variedades;

AQ é a área total destinada ao plantio de cana que será colhida manualmente;

$\mathrm{P}$ é a quantidade mínima estabelecida para o POL da cana (t);

$\mathrm{P}_{\mathrm{i}}$ é a estimativa de produção de sacarose da variedade $\mathrm{i}\left(\mathrm{t} \mathrm{ha}^{-1}\right)$;

FI e FS são as quantidades mínima e máxima estabelecidas para a fibra da cana (t);

$\mathrm{F}_{\mathrm{i}}$ é a estimativa de fibra da variedade $\mathrm{i}\left(\mathrm{t} \mathrm{ha}^{-1}\right)$;

V é a área máxima que cada variedade pode ser plantada.

A restrição (2.1.1.3) garante que toda área destinada para o plantio é usada, a restrição (2.1.1.4) garante a demanda de POL da usina, (2.1.1.5) garante que seja produzida a quantidade de fibra requerida pela usina e as restrições (2.1.1.6) limitam a área de plantio das variedades.

\subsubsection{Modelo para cana com colheita mecanizada}

Denotando CMi o custo de colheita por hectare da variedade i a ser colhida mecanicamente, CMi é calculado da seguinte forma:

$$
\mathrm{CM}_{\mathrm{i}}=\mathrm{C}_{\mathrm{coi}}+\mathrm{C}_{\mathrm{ti}}
$$

sendo: $\mathrm{C}_{\text {coi }}$ é o custo do corte da cana de variedade $\mathrm{i}\left(\mathrm{R} \$ \mathrm{ha}^{-1}\right)$;

$\mathrm{C}_{\mathrm{ti}}$ é o custo de transporte da cana de variedade $\mathrm{i}\left(\mathrm{R} \$ \mathrm{ha}^{-1}\right)$.

Denotando por $\mathrm{X}_{\mathrm{i}}$ a variável do modelo que fornecerá a área (ha) em que deverá ser plantada a cana de variedade i, propõe-se o modelo:

$$
\min \sum_{i=1}^{n} C M_{i} X_{i}
$$

sujeito a 


$$
\begin{aligned}
& \sum_{i=1}^{n} X_{i}=A M \\
& \sum_{i=1}^{n} P_{i} X_{i} \geq P \\
& F I \leq \sum_{i=1}^{n} F_{i} X_{i} \leq F S \\
& 0 \leq X_{i} \leq V, \mathrm{i}=1, \ldots, \mathrm{n} .
\end{aligned}
$$

onde,

$\mathrm{i}=1,2, \ldots, \mathrm{n}$ são os índices que representam as variedades;

AM é a área total destinada ao plantio de cana que será colhida mecanicamente;

$\mathrm{P}$ é a quantidade mínima estabelecida para o POL da cana (t);

$\mathrm{P}_{\mathrm{i}}$ é a estimativa de produção de sacarose da variedade $\mathrm{i}\left(\mathrm{t} \mathrm{ha}^{-1}\right)$;

FI e FS são as quantidades mínima e máxima estabelecidas para a fibra da cana (t);

$\mathrm{F}_{\mathrm{i}}$ é a estimativa de fibra da variedade $\mathrm{i}\left(\mathrm{t} \mathrm{ha}^{-1}\right)$;

V é a área máxima que cada variedade pode ser plantada.

A restrição (2.1.2.3) garante que toda área destinada para o plantio é usada, a restrição (2.1.2.4) garante a demanda de POL da usina, (2.1.2.5) garante que seja produzida a quantidade de fibra requerida pela usina e as restrições (2.1.2.6) limitam a área de plantio das variedades.

As formulações dos modelos dos itens 2.1.1 e 2.1.2 são modelos de programação linear, que podem ser resolvidos por qualquer software de otimização linear, inclusive utilizando a ferramenta Solver da planilha Excel. Esses modelos também podem ser resolvidos pelo método simplex.

\section{RESULTADOS E DISCUSSÃO}

Neste capítulo é apresentada uma aplicação da planilha desenvolvida para resolver o modelo dos itens 2.1.1 e 2.1.2. Esta contém duas interfaces: uma de entrada e outra de saída. Os dados de entrada são: área total para o plantio, áreas de colheita manual e mecanizada e as variedades que são adaptáveis ao solo.

Conforme visualizado na Figura 1, deve-se colocar 1 se a variedade é adaptável ao solo e 0 , em caso contrário. 


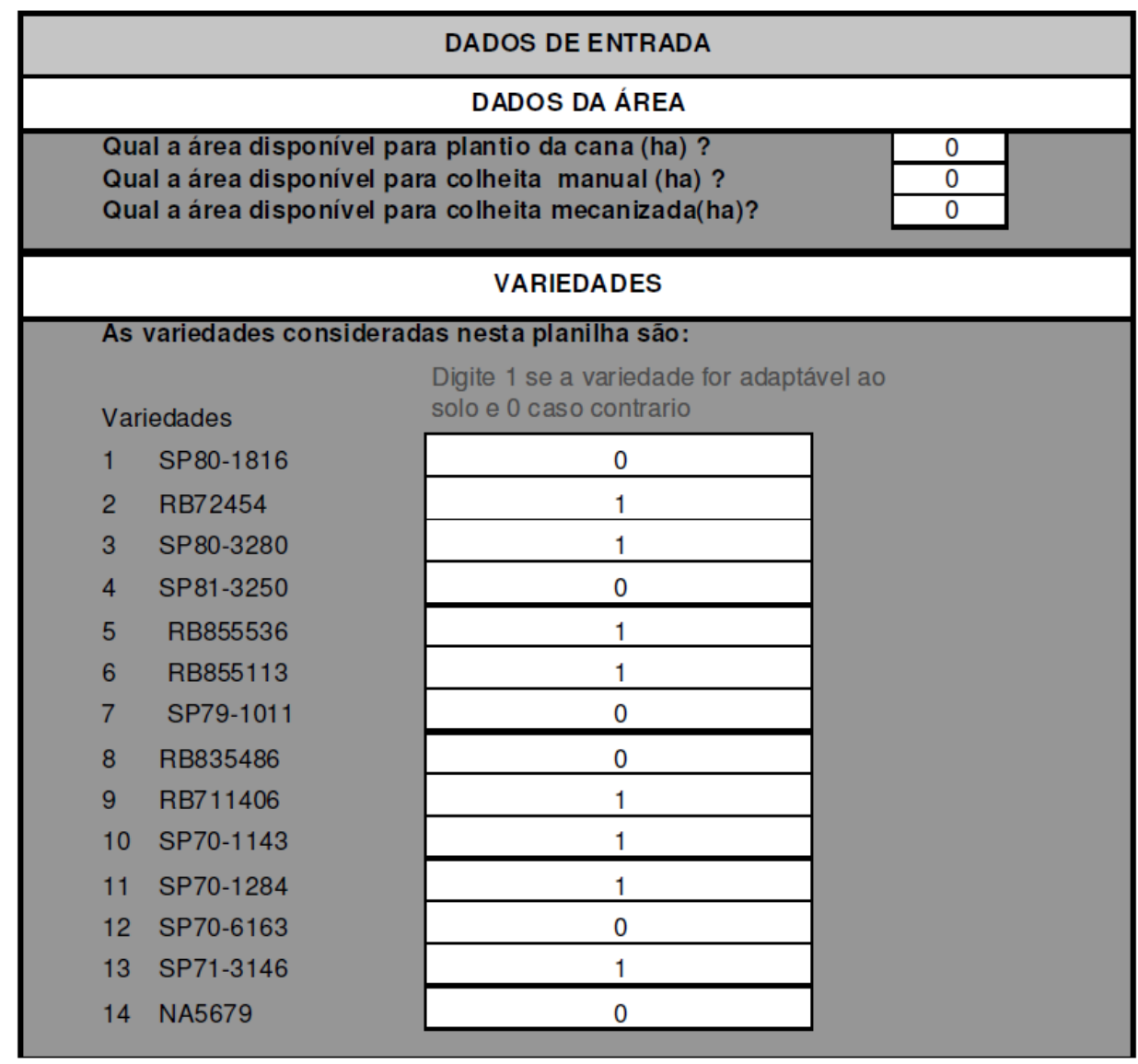

Figura 1 - Interface de entrada da planilha, onde são inseridos os dados.

A planilha contém algumas das variedades mais utilizadas no estado de São Paulo, mas a qualquer momento pode ser facilmente modificadas ou inseridas novas variedades, desde que se conheça os parâmetros necessários para implementação do modelo, conforme a Tabela 1.

Com os dados de entrada, o modelo de programação linear é executado e automaticamente são gerados os dados de saída.

Os dados de saída são: a área que cada variedade deve ser plantada, as estimativas dos custos por operação e total, gastos de energia por operação e do balanço energético, conforme demonstrado na Figura 2. 


\begin{tabular}{|c|c|c|c|c|}
\hline \multicolumn{5}{|c|}{ DADOS DE SAÍDA } \\
\hline \multicolumn{5}{|c|}{ ÁREA DE CADA VARIEDADE A SER PLANTADA (ha) } \\
\hline \multicolumn{2}{|c|}{ Variedades } & \multicolumn{2}{|c|}{ Colheita $m$ anual } & Colheita mecanizada \\
\hline & SP $80-1816$ & \multicolumn{2}{|c|}{0,00} & 0,00 \\
\hline 2 & RB72454 & \multicolumn{2}{|c|}{0,00} & 0,00 \\
\hline 3 & SP $80-3280$ & \multicolumn{2}{|c|}{0,00} & 0,00 \\
\hline & SP $81-3250$ & \multicolumn{2}{|c|}{0,00} & 0,00 \\
\hline & RB 855536 & \multicolumn{2}{|c|}{0,00} & 0,00 \\
\hline & RB 855113 & \multicolumn{2}{|c|}{0,00} & 0,00 \\
\hline & SP79-1011 & \multicolumn{2}{|c|}{0,00} & 0,00 \\
\hline 8 & RB835486 & \multicolumn{2}{|c|}{0,00} & 0,00 \\
\hline & RB711406 & \multicolumn{2}{|c|}{0,00} & 0,00 \\
\hline \multicolumn{2}{|c|}{$10 \quad S P 70-1143$} & \multicolumn{2}{|c|}{0,00} & 12,91 \\
\hline \multicolumn{5}{|c|}{ CUSTOS POR OPERAÇÃO (REAIS) } \\
\hline \multirow{5}{*}{\multicolumn{2}{|c|}{$\begin{array}{l}\text { PREPARO DE SOLO } \\
\text { PLANTIO } \\
\text { COLHEITA } \\
\text { PALHICCO } \\
\text { TOTAL }\end{array}$}} & \multirow{5}{*}{$\begin{array}{l}R \$ \\
R \$ \\
R \$ \\
R \$ \\
R \$\end{array}$} & 0,00 & \multirow[b]{2}{*}{ Manual Mecanizada } \\
\hline & & & 0,00 & \\
\hline & & & 0,00 & \multirow[t]{2}{*}{\begin{tabular}{l|l}
0,00 & 0,00 \\
\end{tabular}} \\
\hline & & & 0,00 & \\
\hline & & & 0,00 & \\
\hline \multicolumn{5}{|c|}{ GASTO DE ENREGIA POR OPERAÇÃO (M J) } \\
\hline \multirow{5}{*}{\multicolumn{2}{|c|}{$\begin{array}{l}\text { PREPARO DE SOLO } \\
\text { PLANTIO } \\
\text { COLHEITA } \\
\text { PALHICLO } \\
\text { TOTAL }\end{array}$}} & & 0,00 & \\
\hline & & MJ & 0,00 & Mecanizada \\
\hline & & MJ & 0,00 & \begin{tabular}{r|r}
0,00 & 0,00 \\
\end{tabular} \\
\hline & & mJ & 0,00 & \\
\hline & & & 0,00 & \\
\hline & & BALA & NREG & IA (M J) \\
\hline BAL & ANÇO DE ENER & & 0,00 & \\
\hline
\end{tabular}

Figura 2 - Interface da saída de dados.

A planilha foi aplicada utilizando dados de uma usina da região de Taquarituba/SP conforme descrito por Lima (2009). Trabalhou-se, portanto, com as seguintes variedades adaptáveis ao solo da referida região: SP80-1846, RB72454, SP80-3280, SP81-3250, RB855536, RB855113, SP79-1011, RB835486, RB711406 e SP70-1143.

$\mathrm{Na}$ Tabela 1 estão apresentados os dados das variedades, necessários para a aplicação do modelo, a saber que $\mathrm{i}=$ índice associado as variedades; $\mathrm{V}_{\mathrm{i}}=$ estimativa do volume do palhiço produzido em uma tonelada da variedade $\mathrm{i}$ (obtido a partir da massa especifica do palhiço); $\mathrm{P}_{\mathrm{Bi}}=$ produtividade de palhiço da variedade $\mathrm{i} ; \mathrm{Ec}_{\mathrm{Bi}}=$ poder calorífico útil do palhiço produzido pela variedade $\mathrm{i} ; \mathrm{A}_{\mathrm{i}}=$ produtividade de açú- 
car fermentescível (POL) da variedade $\mathrm{i} ; \mathrm{Q}_{\mathrm{i}}=$ estimativa do volume do palhiço por unidade de área plantada da variedade i e $F_{i}=$ produtividade de fibra da variedade $i$.

Tabela 1 - Dados das variedades adaptáveis ao solo da Usina, na região de Taquarituba/SP.

\begin{tabular}{|c|c|c|c|c|c|c|c|c|}
\hline $\mathbf{i}$ & Variedade & $\begin{array}{r}V_{i} \\
m^{3} \cdot t^{-1} \\
\end{array}$ & $\begin{array}{l}\mathbf{P}_{\mathbf{B i}} \\
\text { t.ha }\end{array}$ & $\begin{array}{c}\mathbf{E c}_{\mathrm{Bi}} \\
\text { Mcal.t. }^{-1}\end{array}$ & $\begin{array}{c}A_{i} \\
\text { t.ha }^{-1}\end{array}$ & $\begin{array}{c}\mathbf{Q}_{\mathrm{i}} \\
\mathbf{m}^{3} \cdot \mathbf{h a}^{-1} \\
\end{array}$ & $\begin{array}{c}F_{i} \\
\text { t.ha }^{-1}\end{array}$ & $\begin{array}{c}\text { Produtividade } \\
\text { t.ha }^{-1} \\
\end{array}$ \\
\hline 1 & SP80-1816 & 7,964 & 33,36 & 2671,99 & 16,42 & 354,2 & 13,94 & 100,00 \\
\hline 2 & RB72454 & 8,610 & 37,58 & 2649,95 & 20,40 & 299,28 & 12,9 & 186,00 \\
\hline 3 & SP80-3280 & 9,369 & 36,72 & 2602,14 & 18,46 & 316,18 & 12,63 & 158,00 \\
\hline 4 & SP81-3250 & 10,619 & 34,25 & 1947,85 & 18,38 & 320,85 & 11,32 & 179,00 \\
\hline 5 & RB855536 & 9,78 & 26,43 & 2211,95 & 17,05 & 258,46 & 12,51 & 165,00 \\
\hline 6 & RB855113 & 10,87 & 29,38 & 2310,37 & 17,54 & 319,38 & 10,91 & 155,00 \\
\hline 7 & SP79-1011 & 8,91 & 24,09 & 1977,47 & 15,80 & 214,72 & 10,33 & 158,00 \\
\hline 8 & RB835486 & 9,56 & 21,53 & 2444,2 & 12,84 & 205,77 & 9,28 & 155,00 \\
\hline 9 & RB711406 & 12,32 & 33,20 & 2008,83 & 20,77 & 410,29 & 16,12 & 183,00 \\
\hline 10 & SP70-1143 & 7,05 & 22,14 & 1924,8 & 15,01 & 155,98 & 11,59 & 155,00 \\
\hline
\end{tabular}

Fonte: Lima (2009)

Para ilustração do funcionamento desta planilha, utilizou-se as 10 variedades e uma área de 315,81 hectares, que foi dividida em duas partes, de acordo com a exigência da usina: uma de 100 hectares para a cana colhida manualmente com queima e outra de 215,81 hectares para colheita mecanizada. As entradas destes dados são feita da seguinte forma: Primeiro se insere os dados de área total, em seguida os dados de área destinada à colheita manual e mecanizada e posteriormente de escolhe as variedades adaptáveis, digitando 1 para aquelas que são adaptáveis e 0 em caso contrário (Figura 3). 


\begin{tabular}{|c|c|c|c|}
\hline \multicolumn{4}{|c|}{ DADOS DE ENTRADA } \\
\hline \multicolumn{4}{|c|}{ DADOS DA ÁREA } \\
\hline \multicolumn{3}{|c|}{$\begin{array}{l}\text { Qual a área disponível para plantio da cana (ha) ? } \\
\text { Qual a área disponível para colheita manual (ha) ? } \\
\text { Qual a área disponível para colheita mecanizada(ha)? }\end{array}$} & $\begin{array}{l}315,81 \\
100,00 \\
215,81 \\
\end{array}$ \\
\hline \multicolumn{4}{|c|}{ VARIEDADES } \\
\hline \multicolumn{4}{|c|}{$\begin{array}{l}\text { As variedades consideradas nesta planilha são: } \\
\qquad \begin{array}{l}\text { Digite } 1 \text { se a variedade for adaptável }\end{array}\end{array}$} \\
\hline & SP80-1816 & 1 & \\
\hline 2 & RB72454 & 1 & \\
\hline 3 & SP80-3280 & 1 & \\
\hline 4 & SP81-3250 & 1 & \\
\hline 5 & RB855536 & 1 & \\
\hline 6 & RB855113 & 1 & \\
\hline 7 & SP79-1011 & 1 & \\
\hline 8 & RB835486 & 1 & \\
\hline 9 & RB711406 & 1 & \\
\hline & SP70-1143 & 1 & \\
\hline & SP70-1284 & 0 & \\
\hline & SP70-6163 & 0 & \\
\hline & SP71-3146 & 0 & \\
\hline & NA5679 & 0 & \\
\hline
\end{tabular}

Figura 3 - Interface de entrada da planilha com os dados inseridos.

Os modelos dos itens 2.1.1 e 2.1.2 determinaram as variedades a serem plantadas para cada área, de forma que os custos fossem mínimos, atendendo às restrições impostas. Observa-se na interface de saída (Figura 4), que para a área com colheita manual, o modelo determinou três variedades, SP80-1816 com 50,0 há; SP80-3280 com 31,2 ha e RB855113 com 18,8 há. Para a área de colheita mecanizada, foram determinadas quatro variedades, a saber: SP80-1816, com uma área de 71,9 há; SP80-3280 com área de 59,0 ha; RB855536 com 71,9 ha e SP70-1143 com 12,9 ha. 


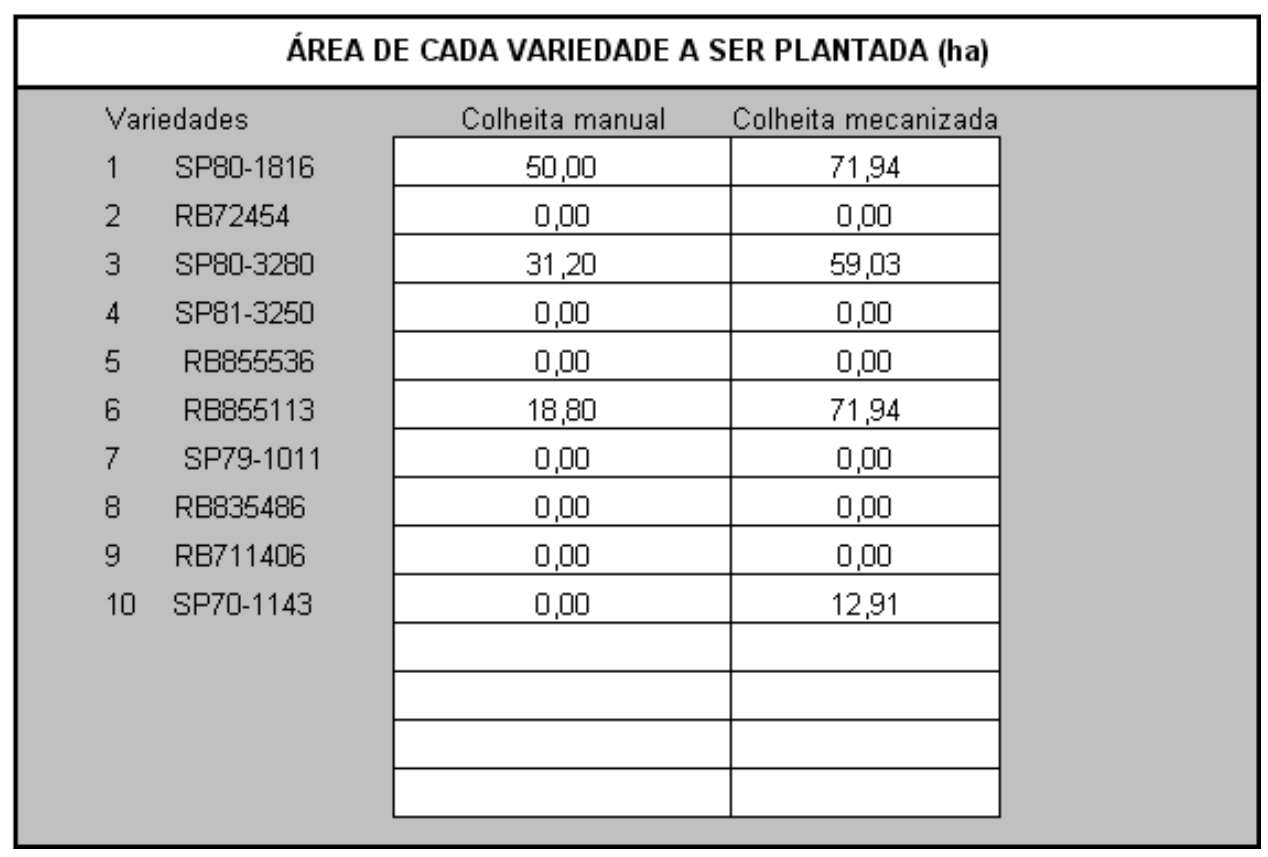

Figura 4 - Resultado da aplicação dos modelos dos itens (2.1.1 e 2.1.2).

A partir do resultado do modelo, a planilha desenvolvida calcula automaticamente as estimativas de custos para produção e entrega da cana-de-açúcar na indústria. Na Figura 5 estão apresentados os resultados gerados pela planilha para os custos de produção da cana-de-açúcar para uma área de 315,81 hectares.

\section{CUSTOS POR OPERAÇÃO (REAIS)}

PREPARO DE SOLO

PLANTIO

COLHEITA

PALHIÇO

TOTAL

\begin{tabular}{|c|c|c|c|}
\hline $\mathrm{R} \$$ & $163.763,28$ & & \\
\hline $\mathrm{R} \$$ & $897.115,15$ & Manual & Mecanizada \\
\hline $\mathrm{RS}$ & $690.461,70$ & $188.426,31$ & $502.035,40$ \\
\hline $\mathrm{R} \$$ & $94.362,92$ & & \\
\hline $\mathrm{RS}$ & $1.845 .703,05$ & & \\
\hline
\end{tabular}

Figura 5 - Custo de produção da cana-de-açúcar para a área de 315,81 hectares.

Como demonstrado na Figura 5, o custo total para a produção da cultura da cana-de-açúcar para esta referida área foi de $\mathrm{R} \$ 1.845 .703,05$, estimando, portanto, um custo de $\mathrm{R} \$ 5.982,78$ por hectare. $\mathrm{O}$ custo de colheita foi de $\mathrm{R} \$ 690.416,70$, que é a somatória da colheita manual $(\mathrm{R} \$ 188.426,31)$ e colheita 
mecanizada ( $\mathrm{R} \$ 502.035,40)$. Neste caso, a colheita mecanizada apresentou maior custo por ser uma operação totalmente mecanizada, ou seja, com maior consumo de combustível.

Na Figura 6, são apresentados os custos separados por operação em porcentagem. O plantio representou a maior participação nos custos, com $48,61 \%$, seguida da colheita com $37,41 \%$, do preparo de solo com $8,87 \%$ e do recolhimento do palhiço com $5,11 \%$ do custo total de produção.

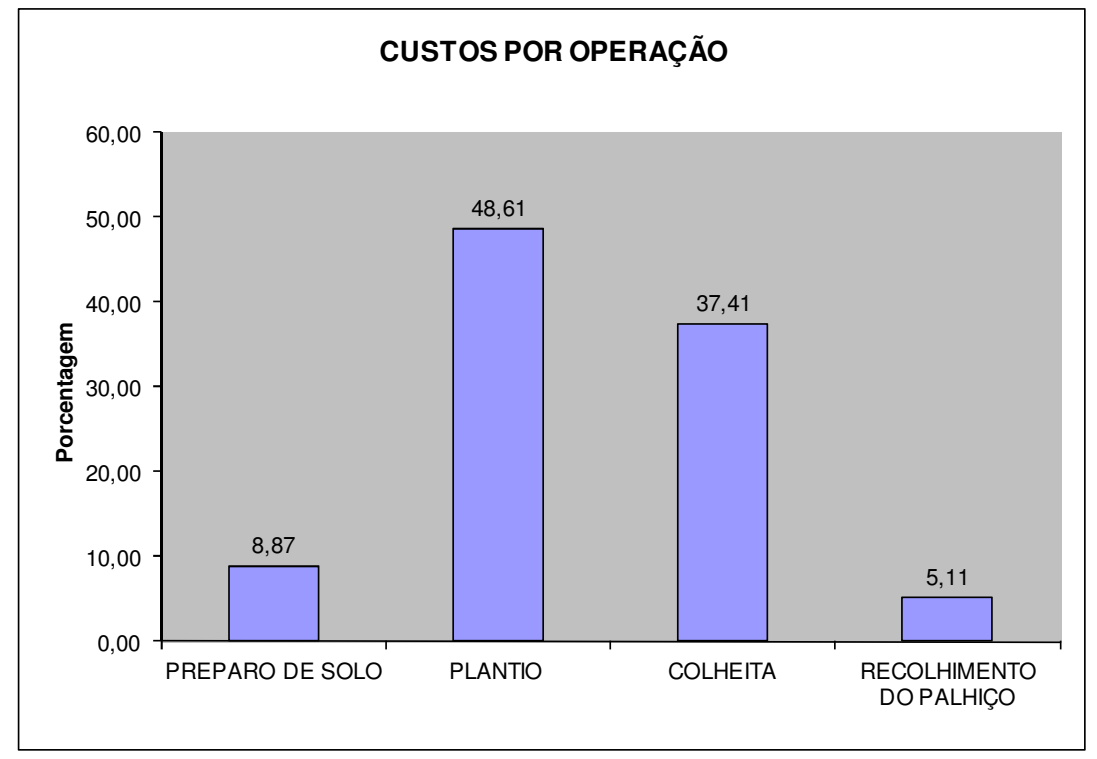

Figura 6 - Custo de operações em porcentagem da produção de cana.

Na Figura 7 são apresentadas resumidamente as estimativas do gasto de energia não renovável (consumo de combustível) por etapa de produção da cana-de-açúcar, para uma área estimada de 315,81 hectares.

\begin{tabular}{|c|c|c|c|c|}
\hline \multicolumn{5}{|c|}{ GASTO DE ENERGIA POR OPERAÇÄO (MJ) } \\
\hline PREPARO DE SOLO & MJ & $1.808 .326,85$ & & \\
\hline PLANTIO & MJ & $1.096 .574,90$ & Manual & Mecanizada \\
\hline COLHEITA & MJ & $1.816 .256,51$ & $246.776,47$ & $1.569 .480,04$ \\
\hline PALHIÇO & MJ & $456.647,98$ & & \\
\hline TOTAL & MJ & $5.177 .806,24$ & & \\
\hline
\end{tabular}

Figura 7 - Total de energia não renovável gasta por operação. 
Como demonstrado na Figura 7, a estimativa do consumo de energia não renovável foi de 5.176.776,74 MJ. O preparo de solo apresentou maior consumo de energia não renovável, seguido da colheita mecanizada. A operação de colheita manual foi a que apresentou o menor gasto de energia não renovável com consumo estimado de 246.776,47 MJ.

$\mathrm{Na}$ Figura 8 pode-se observar que a operação de colheita envolve o maior consumo de energia, $35,08 \%$ do total, seguida pela operação de preparo de solo com $34,92 \%$, plantio com $21,18 \%$ e recolhimento do palhiço com $8,82 \%$ do gasto total de energia.

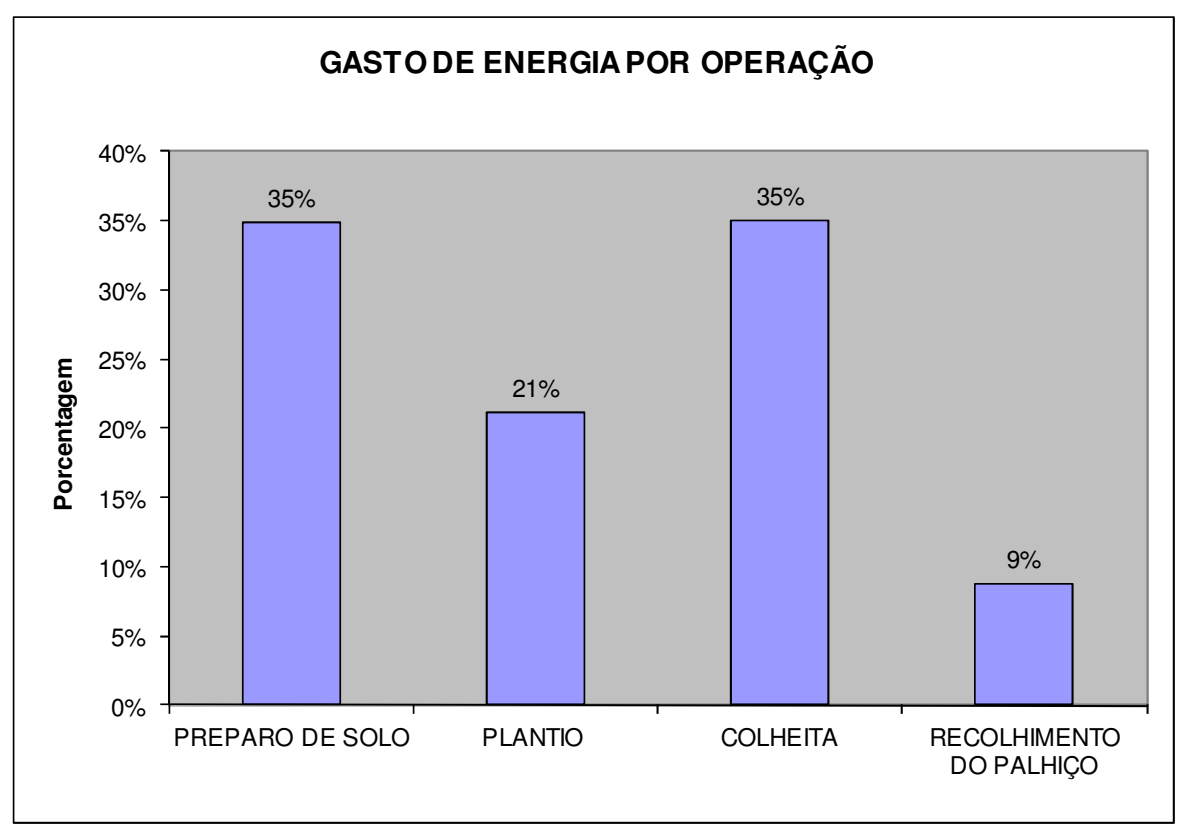

Figura 8 - Gasto de energia não renovável por operação.

A energia estimada gerada pela queima do palhiço foi de 17.485.583,23 MJ. Portanto o balanço de energia foi de 12.308.806,49 MJ, conforme determinado na planilha, e visualizado na Figura 9.

\section{BALANÇO DE ENERGIA (MJ)}

BALANÇO DE ENERGIA $12.308 .806,49$

Figura 9 - Balanço de energia não renovável. 


\section{CONCLUSÕES}

As investigações feitas durante o desenvolvimento deste projeto mostraram que as empresas sucroalcooleiras têm crescido em um ritmo muito acelerado. Com as grandes dimensões atualmente, destas empresas, o gerenciamento deste setor tem sido cada dia mais complexo, havendo necessidade de lançar mão de ferramentas que auxiliem nas tomadas de decisões. Assim, as técnicas matemáticas e computacionais têm sido um instrumento de grande auxílio. O modelo de programação linear proposto para minimização de custos, juntamente com a planilha implementada neste trabalho, se mostram ferramentas promissoras para esta finalidade.

\section{AGRADECIMENTOS}

A FAPESP e CAPES, pelo apoio financeiro ao projeto desta linha de pesquisa.

\section{REFERÊNCIAS}

\section{FLORENTINO, H.O. Programação linear inteira em problemas de aproveitamento da biomassa} residual de colheita da cana-de-açúcar. 2005. 65f. Tese (Livre Docência) Faculdade de Ciências Agronômicas, Universidade Estadual Paulista, Botucatu, 2005.

FLORENTINO, H.O.; MORENO, E.V.; SARTORI, M.M.P. Multiobjective optimization of economic balances of sugarcane harvest biomass. Scientia Agrícola, v. 65, p. 561-564, 2008.

LIMA, A.D. de. Otimização do aproveitamento do palhiço da cana-de-açúcar. 2009. 76f. Tese (Doutorado em Agronomia/ Energia na Agricultura) - Faculdade de Ciências Agronômicas, Universidade Estadual Paulista Botucatu, 2009.

MARQUES, P.V. (Coord.). Custo de produção agrícola e industrial de açúcar e álcool no Brasil na safra 2007/2008. Piracicaba: Universidade de São Paulo, Escola Superior de Agricultura "Luiz de Queiroz”, Programa de Educação Continuada em Economia e Gestão de Empresas/Departamento de Economia, Administração e Sociologia, 2009. 194 p. Relatório apresentado a Confederação da Agricultura e Pecuária do Brasil - CNA. 
TOLENTINO, G.; FLORENTINO, H.O. Programação linear inteira aplicada ao aproveitamento do palhiço da cana-de-açúcar. Energia na Agricultura, v. 23, p. 13-33, 2008.

UNIVERSIDADE DE SÃO PAULO. Vendas do setor sucroenergético representam 1,5\% do PIB, diz estudo. 2009. Disponível em:<http://www4.usp.br/index.php/meio-ambiente/17953-vendas-do-setorsucroenergetico-representam-15-do-pib-diz-estudo>. Acesso em: 31 maio 2010. 